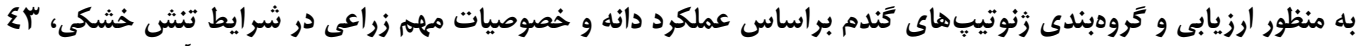

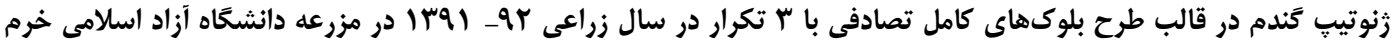

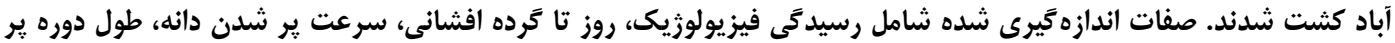

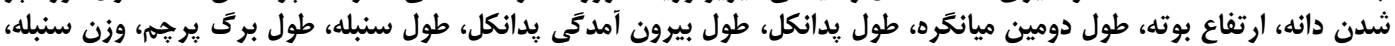

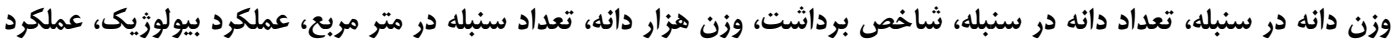

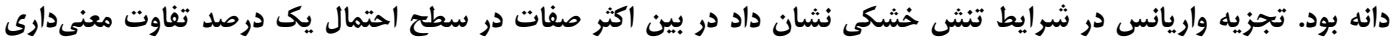

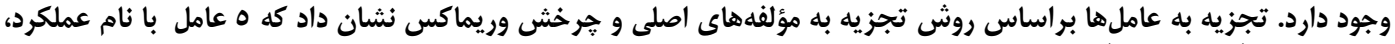

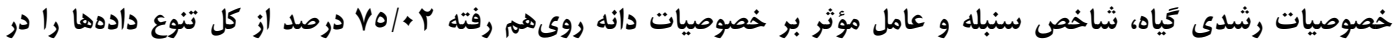

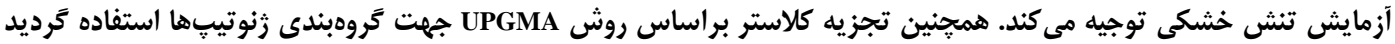

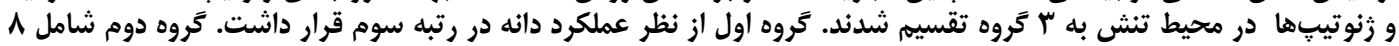

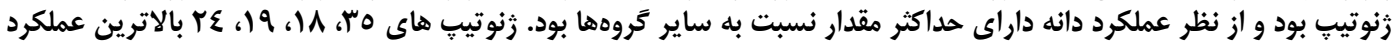

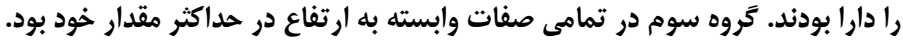

وازههاى كليدى: تجزيه به عاملها، تجزيه كلاستر، تنث خشكى، كَندم

يروتئينهاى شوى حرارتى و تبعيض ايزوتوٍ كربن انجام

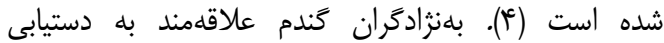

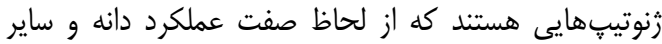

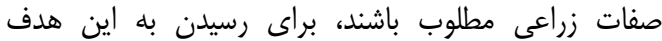

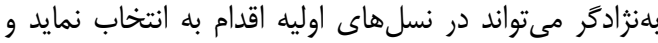

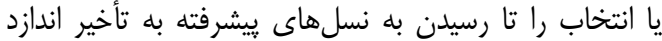
شاخص

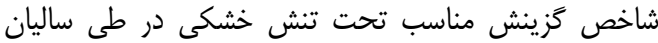

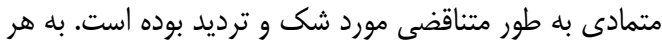

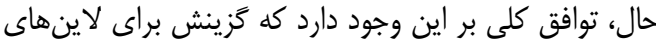

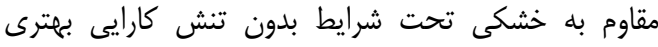

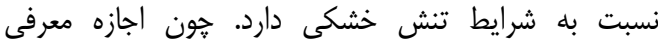

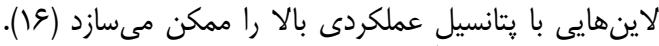

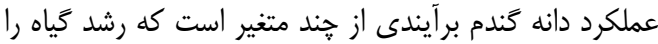

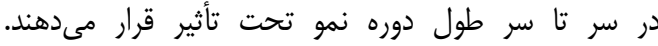

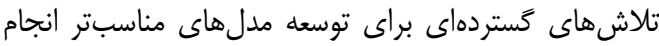

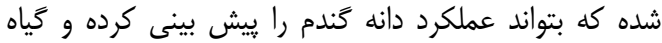

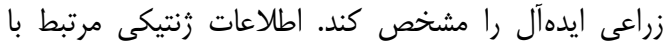

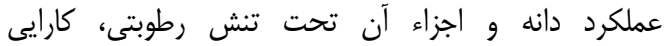

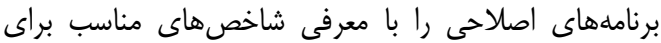

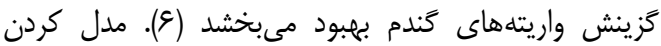

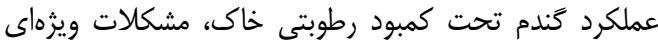

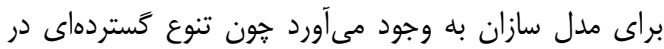

گَندم يكى از مهمترين محصولات زراعى از لحاظ سطح

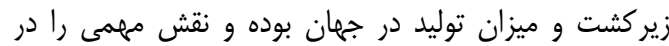

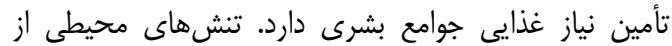

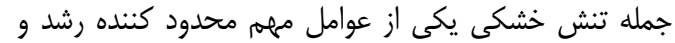

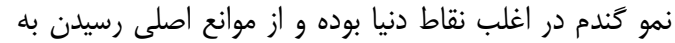

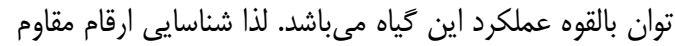

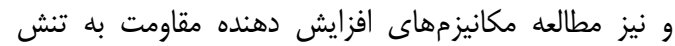

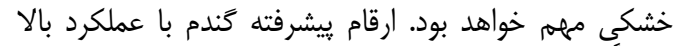

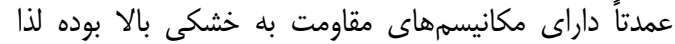

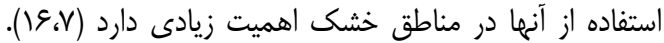

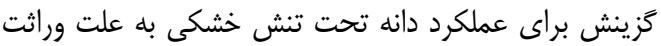

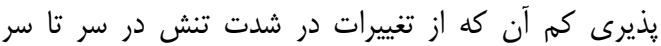

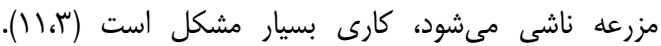

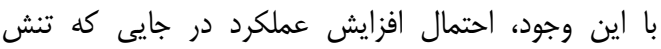

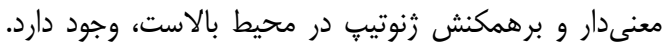

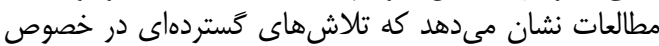

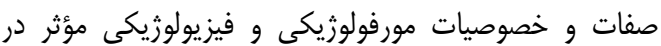

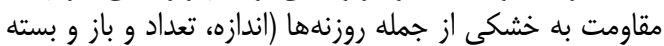

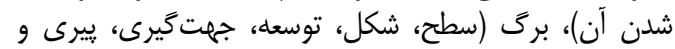

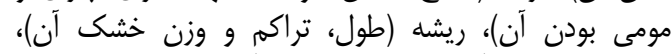

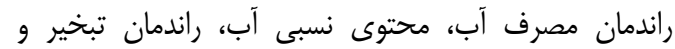

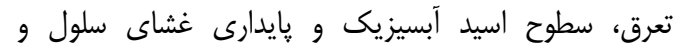


دانه در سنبله، وزن هزار دانه، تعداد سنبله در متر مربع، طول

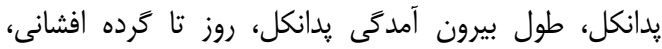

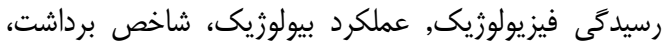

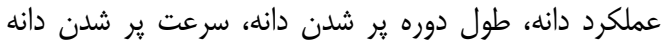

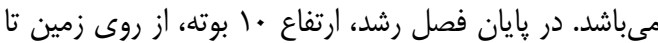

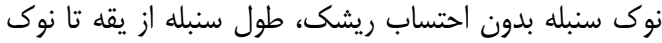

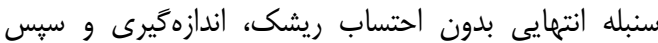

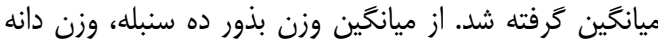

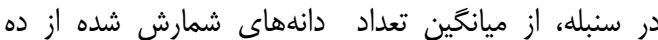

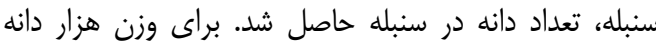

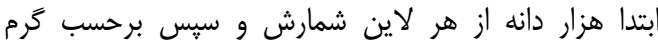

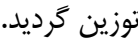

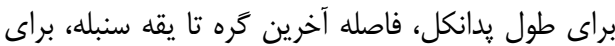

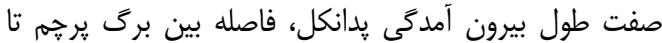

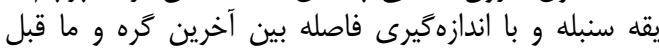

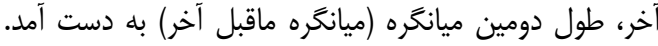

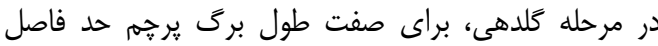

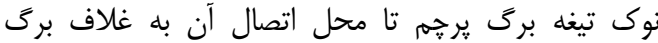

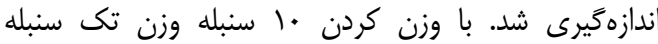

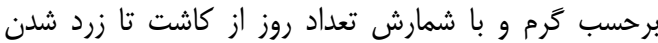

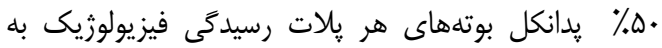

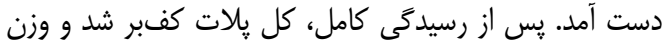

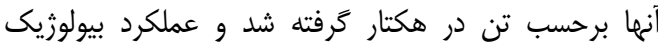

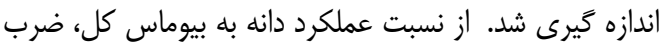

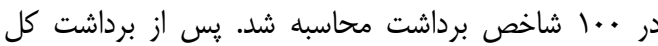

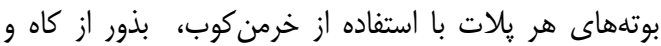

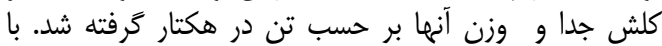

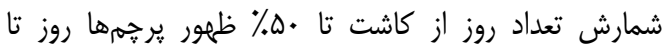

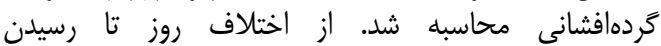

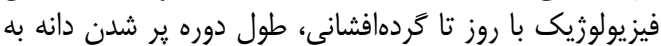

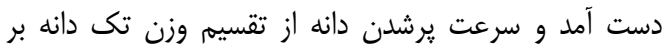

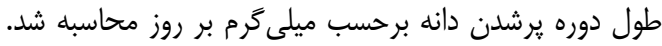

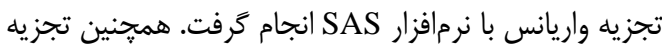

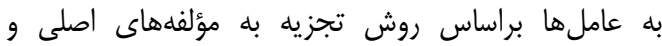

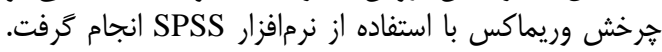

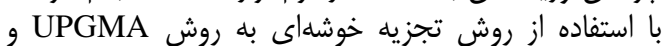

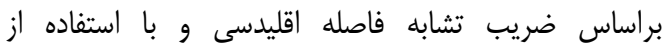

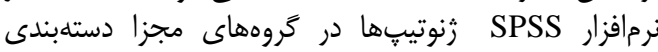

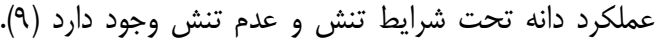

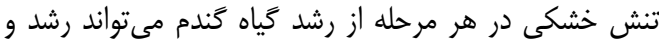

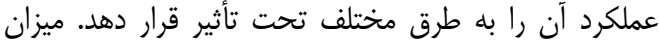

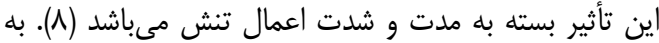

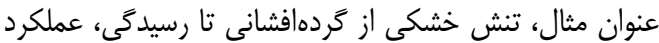

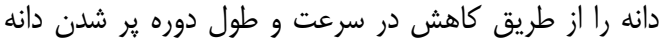

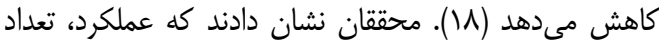

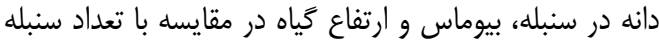

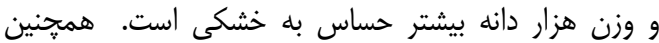

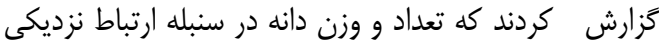

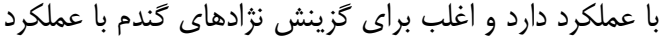

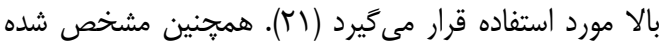

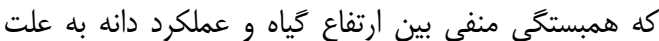

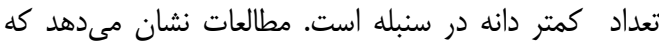

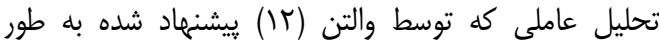

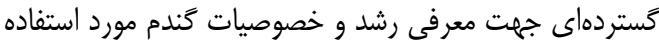

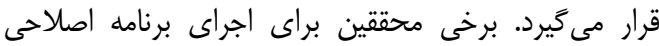

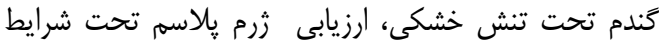

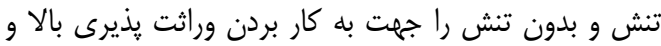

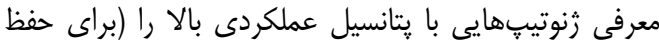

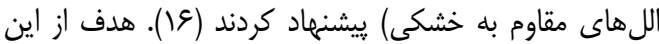

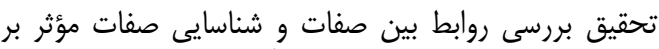

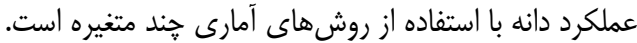

\section{مواد و روشها}

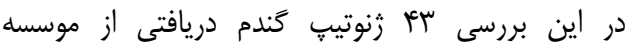

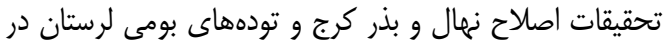

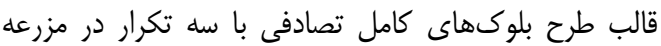

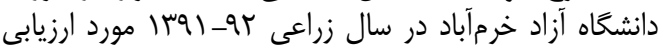

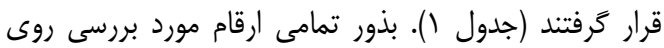

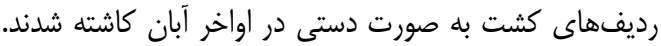

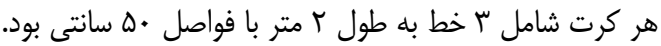

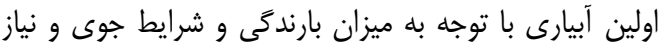

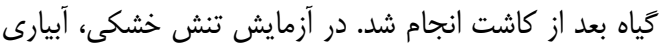

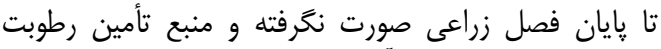

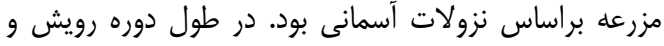

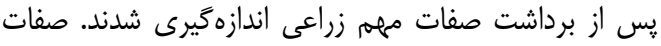

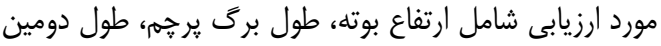

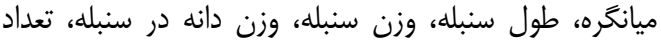


Table 1. Name wheat genotypes used in trial

جدول ا- اسامى زنوتيڤهاى مورد استفاده در آزمايش

\begin{tabular}{|c|c|c|c|c|c|c|c|c|}
\hline شماره & رنوتيب & تيٍ رشد & شماره & رنونيّ & تيڤ رشد & شماره & 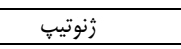 & تيڤ رشد \\
\hline 1 & سيوند & بهاره & 19 & بيشتاز & بعاره & r & NESTOR & بهاره \\
\hline r & سيستان & بهاره & IV & 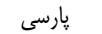 & بهاره & זr & FLORKAWA & بهاره \\
\hline r & سيروان & بهاره & 11 & بهار & بهاره & זץ & IRENA & بهاره \\
\hline i & 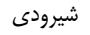 & بهاره & 19 & بم & بهاره & mf & CHEN/AGILOPS & بهاره \\
\hline$\Delta$ & شيراز & بجاره & r. & 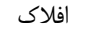 & بهاره & ra & SERI/PAYON & بهاره | بهار \\
\hline 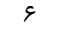 & مرودشت & بهاره & r) & 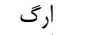 & بهاره & צ & SITA/CHIL & بهاره \\
\hline $\mathrm{v}$ & 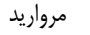 & بهاره & r & آرتا & بجاره & rV & ZEMAMRA-8 & بهاره \\
\hline$\wedge$ & 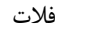 & بهاره & זr & 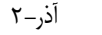 & زمستانه & ץ & BERKUT & بهاره \\
\hline 9 & نيشابور & بهاره & re & سردارى & زمستانه & rq & PIGO & بهاره \\
\hline 1 . & 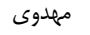 & بمهاره & ro & سيمره & بجاره & f. & SERI & بهاره \\
\hline 11 & سياهان & بهاره & tq & شاهيوندى & بهاره & i) & CROC & بهاره \\
\hline ir & ل & بهاره & tr & زاكرس & بهاره & ft & BAVICOVA & بهاره \\
\hline rו & دريا & بهاره & r & 5 & بهاره & Tr & SERI-82 & بهاره \\
\hline lif & داراب-r & بهاره & זq & مارون & يهاره & & & \\
\hline 10 & 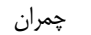 & بهاره & r. & بإستور & بهاره & & & \\
\hline
\end{tabular}

ا داراى بيشترين ميانگين در ميان ساير زنوتيڤها بود.

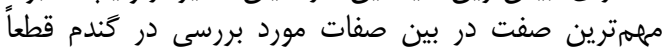

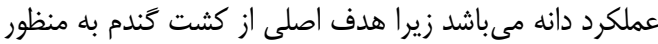

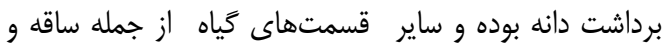

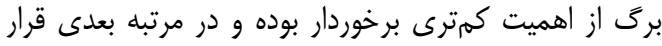

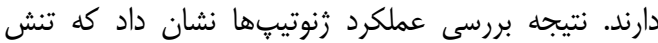

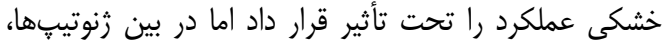

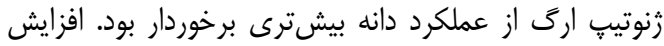

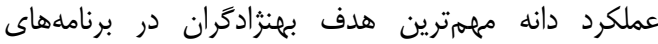

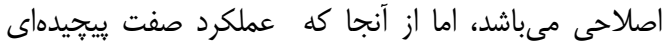

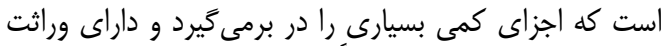

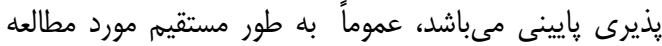

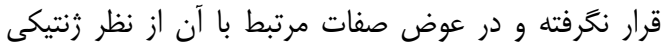

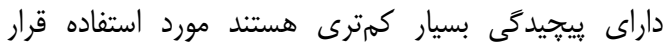

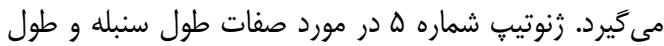

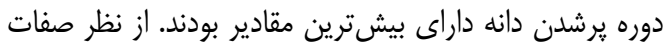

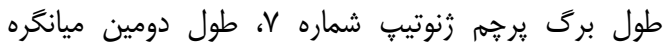

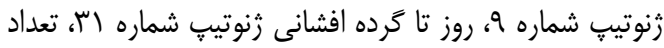

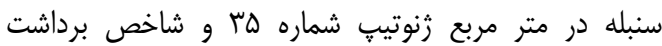

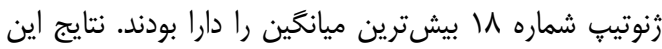

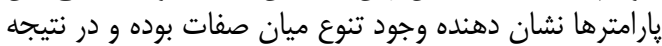

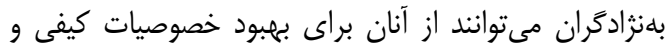

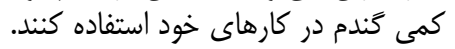

نتايج و بحث

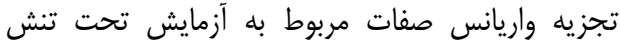

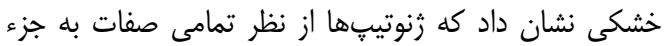

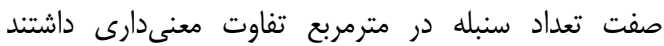

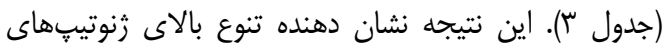

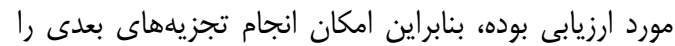

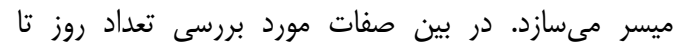

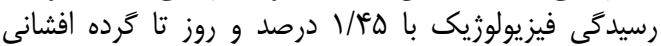

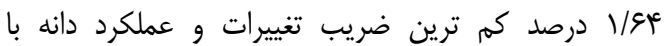

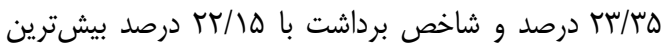

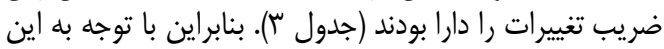

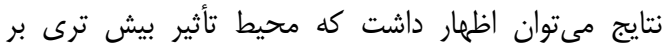

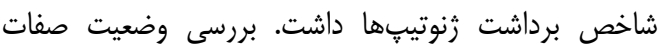

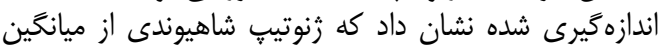

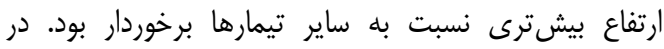

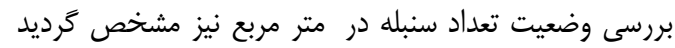

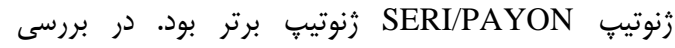

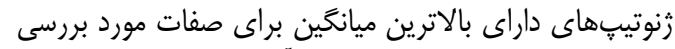

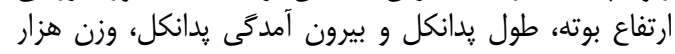

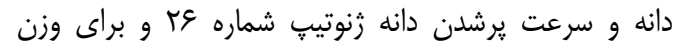
سنبله، وزن دانه در سنبله

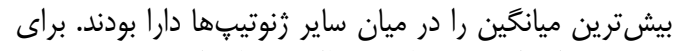
صفات تعداد دانه در سنبله و عملكرد بيولوزيك ريك زنوتيني شماره

Table 2. The Max and Min of traits

\begin{tabular}{|c|c|c|c|c|c|c|c|c|c|}
\hline شماره & حداقل & شماره & حداكثر & صفات & شماره & حداقل & شماره & حداكثر & صفات \\
\hline Tr & $99 / 14$ & Te & NV/KT & وزن سنبله (كرم) & $T F^{c}$ & I/VT & r) & $r / \mathrm{N}$ & ر رتفاع بوته (سانتىمتر) \\
\hline rq & $\mathrm{V} / \mathrm{VV}$ & a & $11 / 91$ & تعداد دانه در سنبله & re & Tr/q9 & 1 & 99 & طول سنبله (سانتىمتر) \\
\hline f & $|0 / 9|$ & Q & $\mathrm{rq} / \mathrm{Av}$ & وزن دانه در سنبله (كرم) & t & I/VT & r) & $r / v q$ & طول پدانكل (سانتىمتر) \\
\hline f & $\Delta / \mp \Delta$ & re & $r T / Q V$ & وزن هزار دانه (كرم) & $v$ & $\mu \varphi / \Delta$. & צ & $\Delta \mathrm{V} / \mathrm{V} \Delta$ & طول بيرون آمدگى پِدانكل (سانتىمتر) \\
\hline Tr & $1 \pi / 9$. & $q$ & W/TK & طول دوره پرشدن دانه & tr & Q) & $\Delta$ & st & طول ميانكره دوم (سانتىمتر) \\
\hline $\mathrm{v}$ & سיץ/19 & ו & gra & سرعت پرشدن دانه & $\mathrm{v}$ & . & q & $1 / .1$ & تعداد روز تا كرده افشانى \\
\hline TV & IVE & $\Delta$ & $\mid \Lambda \Delta / \pi \mu$ & عملكرد دانه (تن) & זו & $1 / 14$ & r & $r / 9 V$ & نعداد روز تا رسيدگى فيزيولوزيك \\
\hline Q & $\| \mathrm{T} / \mathrm{VV}$ & ra & MTr & عملكرد بيولوزيك (تن) & m & $\Delta / 1$. & 1 & $\mid r / \% \Delta$ & تعداد سنبله در متر مربع \\
\hline re & $\mathrm{V} / \mathrm{V}$ & $\mathrm{v}$ & $|f / \Delta|$ & شاخص برداشت & 1 & $19 / 19$ & 11 & re/4a & طول برگ ير جم (سانتىمتر) \\
\hline
\end{tabular}



ارزيابى گֶندين صفات مختلف زراعى در زنوتيڤهاى گندم تحت شرايط تنش خشكى با استفاده از ..

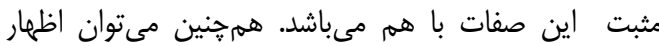

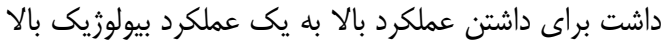

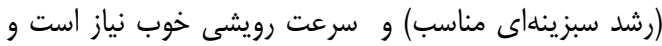

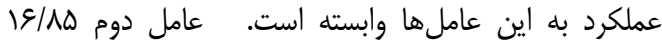

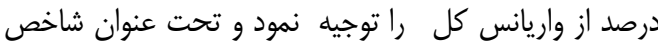

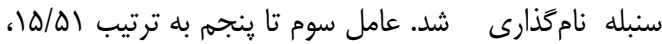

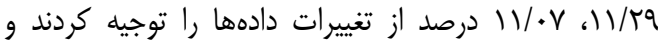

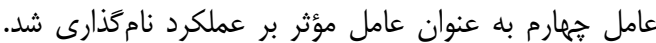

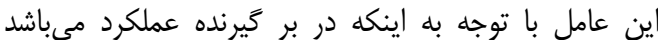

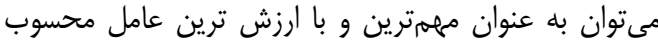

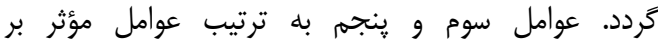

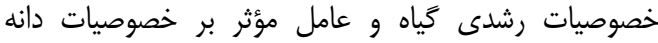

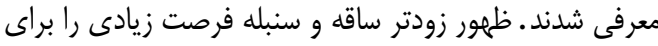

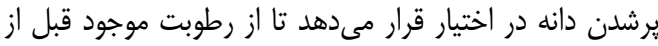

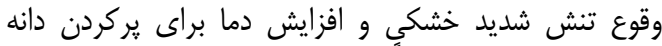

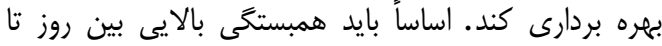

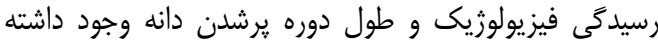

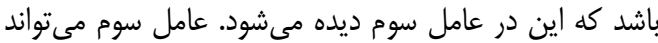

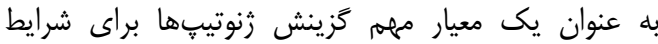

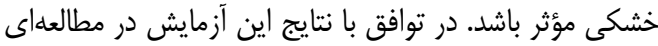

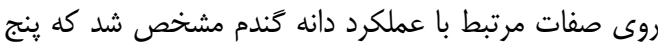

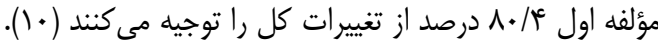

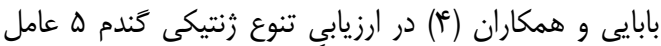

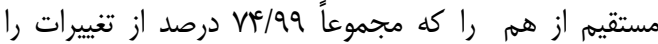

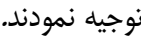

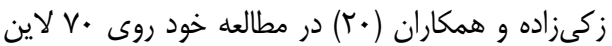

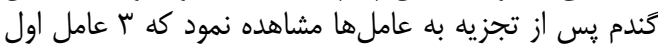

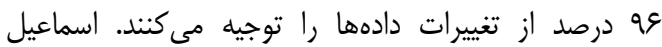

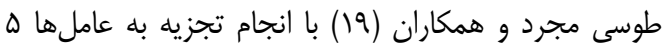

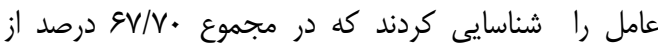

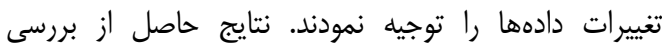

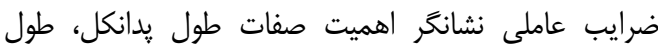

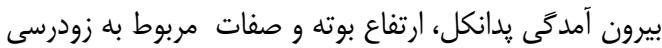

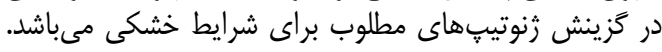

از تجزيه به عاملها در تعيين ارتباط اجزاى عملكرد،

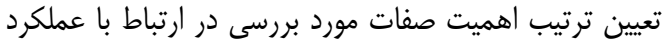

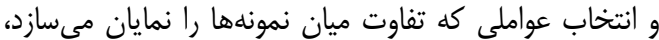
استفاده مى

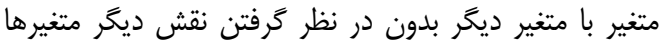

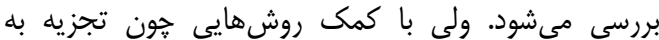

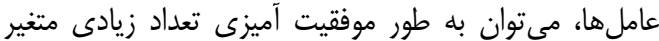

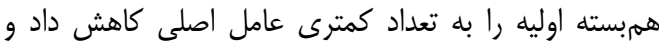

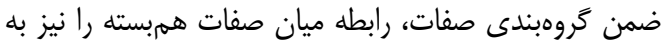

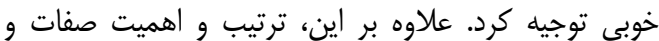

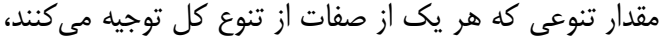

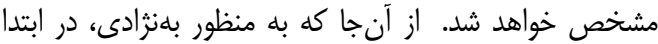

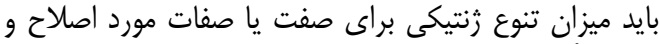

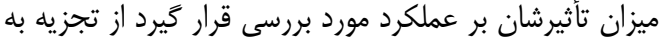

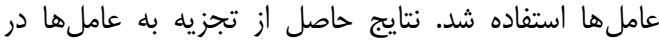

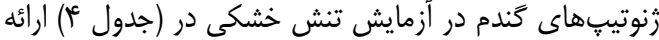

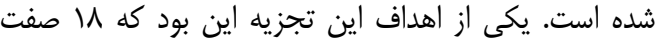

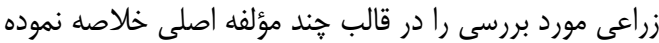

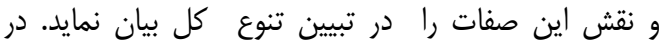

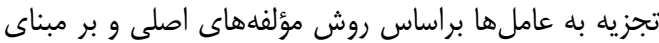

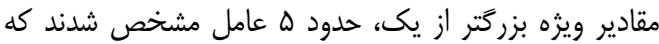

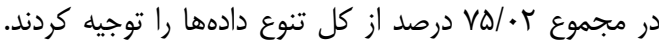

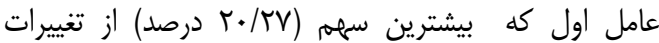

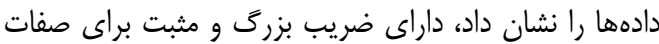

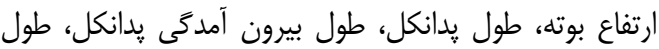

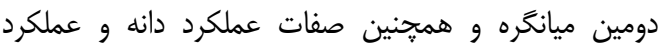

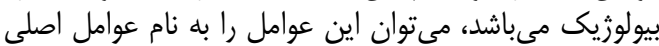

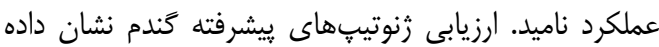

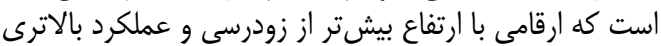

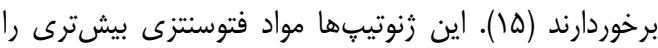

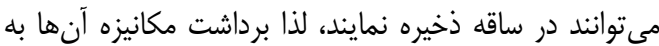

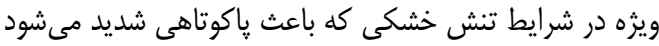

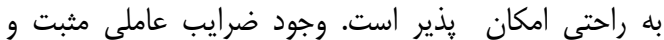
معنىدار براى عملكرد در عامل اول نشاندئ وهنده همبستخى 
Table 3. Variance analysis of wheat of agronomic trais

جدول ب- تجزيه واريانس براى خصوصيات زراعى

\begin{tabular}{|c|c|c|c|c|c|c|c|c|c|}
\hline \multirow{3}{*}{ ضريب تغييرات (٪) } & \multicolumn{3}{|c|}{ ميانگين مربعات } & \multicolumn{5}{|c|}{ ميانكين مربعات } & \multirow{3}{*}{ صفات درجه آزادى } \\
\hline & خطا & تيمار & تكرار & صفات درجه آزادى & ضريب تغييرات (٪) & خطا & تيمار & تكرار & \\
\hline & Af & HT & $r$ & & & $\wedge \leftarrow$ & tet & $r$ & \\
\hline$\% 1 \% / \pi \Delta$ &.$/ 10$ & $\cdot / \mathrm{VA}$ & $\cdot 1 \cdot 0^{\mathrm{nS}}$ & وزن سنبله (كرم) & $\% / v$. & $T \Delta / q D$ & $119 / 4 \Lambda^{\cdots+}$ & $r \Delta \cdot{ }^{*}$ & ارتفاع بوته (سانتى متر) \\
\hline$\% I T / T V$ & rT/qG & $r T / / V^{T *}$ & $V N / r \omega^{n s}$ & تعداد دانه در سنبله & $\% 1 \cdot / 19$ & $1 / I V$ & $r / q \varphi^{-\pi *}$ & $\cdot / 9 \gamma^{\mathrm{ns}}$ & طول برى ير خم( سانتى متر) \\
\hline$\% / \Delta / 9 \Delta$ & .1 .9 &.$/ 40$ & $\cdot / \cdot \Delta^{\mathrm{ns}}$ & وزن دانه در سنبله (خرم) & $\% 11 / 4 r$ & $1 / 10$ & $r / r T^{N \pi}$ & $\cdot / \cdot \varphi^{\mathrm{ns}}$ & طول سنبله (سانتى متر) \\
\hline$\% \cdot N \cdot 1$ & $11 / 89$ & $99 / 19^{* \pi}$ & $r \cdot / v \Lambda^{\mathrm{ns}}$ & وزن هزار دانه (كرم) & $\% 1 \cdot / \Delta$ & $8 / \cdot V$ & $\Delta T / 9)^{\pi * T}$ & $r m / F q^{m-\pi}$ & طول يدانكل (سانتى متر) \\
\hline$\% / 1 \Delta \Lambda$ & $\mid F / \mu$ & $T V / \Delta q^{*}$ & $\mid Q / R V^{\mathrm{ns}}$ & طول دوره بر شدن دانه & $\% N / M F$ & $r / . r$ & TI/AT" & $r .199^{. m}$ & طول بيرون آمدگى بِدانكل (سانتى متر) \\
\hline$\% 1 \cdot / r V$ & $\% \Delta$ & $\cdot / \cdot r^{m *}$ & $\cdot / \cdot 1^{\mathrm{ns}}$ & سرعت ير شدن دانه & $\% ৭ / \wedge f$ & $T / Y)$ & $\mathrm{V} / 90^{-m}$ & $r / 4 r^{n s}$ & طول دومين ميانكره (سانتى متر) \\
\hline D D D &.$/ 49$ & $1 / r^{* * *}$ & $1 / 4 s^{\prime \prime}$ & عملكرد دانه (تن) & $\% 1 / 94$ & $\% / . \Delta$ & $N / 9 q^{* \pi}$ & $+1 / .9^{-m}$ & روز تا كرده افشانى \\
\hline$\% 18 / 94$ & $r / N Q$ & $9 / 9 \varphi^{*-m}$ & $r / q r^{\mathrm{ns}}$ & عملكرد بيولوزيك (تن) & $\% / \uparrow \Delta$ & g/Ar & $\mid V / T V^{m m}$ & $\cdot / 19^{\mathrm{ns}}$ & روز تا رسيدگى فيزيولوزيك \\
\hline$\% \pi / 1 Q$ & $r \Delta / r \Delta$ & $\Delta \mathrm{V} / \cdot \Delta^{\circ}$ & $\Delta F / \Delta Y^{\mathrm{ns}}$ & شاخص برداشت & $\% / r / \wedge r$ & $\mid f \wedge q / 4$. & $r \cdot . q / / r^{n s}$ & $1 \cdot T N / F V^{\mathrm{ns}}$ & تعداد سنبله در متر مربع \\
\hline
\end{tabular}


Table 4. Results of factor analysis

جدول عا- نتايج مربوط به تجزيه عاملها

\begin{tabular}{|c|c|c|c|c|c|}
\hline 1 & t & $r$ & f & $\Delta$ & صفت \\
\hline$\cdot / \Lambda \Delta$ & .1 .9 & $-\cdot / 4 \mid$ & $\cdot / 1 r$ &.$/ \cdot t^{2}$ & ارتفاع(سانتىمتر) \\
\hline$\cdot / \pi V$ &.$/ 10$ & $\cdot / 1$ & - $/ \Delta T$ &.$- / \cdot 1$ & طول برى برجم (سانتىمتر) \\
\hline.$/$. & $\cdot / \pi f$ & $-\cdot / \cdot 1$ & $\overline{. / 4 \Lambda}$ &.$- / 48$ & طول سنبله (سانتىمتر) \\
\hline$\cdot / \Lambda$. & 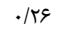 & $\cdot / T^{\prime}$ & -.1 .9 & $\cdot / r \Lambda$ & طول يدانكل (سانتىمتر) \\
\hline$\overline{. / \mathrm{V}}$ &.$/ 14$ &.$/ 1 T$ & $-\cdot / 11$ & 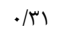 & طول بيرون آمدگى پِدانكل (سانتىمتر) \\
\hline.$/ \mathrm{VQ}$ & $.1 \cdot 1$ & $-\cdot / r \varepsilon$ & . 童 &.$- / .4$ & طول دومين ميانگگره (سانتىمتر) \\
\hline$\overline{. / \cdot 1}$ & -.1 .9 & $-\cdot / \mathrm{VT}$ & $\cdot / T \Delta$ & $\cdot / \cdot v$ & روز تا كردهافشانى \\
\hline$-1 \cdot 0$ &.$/ \mathrm{V}$ & ./Ar & . & -4 & روز تا رسيدگى فيزيولوزيك \\
\hline$\cdot / 1$ & $-\cdot / r V$ & $\overline{-\cdot / r \mid}$ & $\cdot / \kappa$ &.$- / 4 F$ & تعداد سنبله در متر مربع \\
\hline.$/ 1 F$ &.$/ 90$ &.$/ 10$ & $-.1 \cdot 1$ & .1 .9 & وزن سنبله (كَرم) \\
\hline.$/ .4$ &.$/ 9$. &.$/ 1 F$ & $\cdot$ & 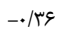 & تعداد دانه در سنبله \\
\hline.$/ 10$ & $\overline{. / 90}$ &.$/ 1 r$ &.$+r$ & .1 .9 & وزن دانه در سنبله (كرم) \\
\hline$\cdot / T \Lambda$ & $\overline{l \cdot r}$ & $-\cdot / \cdot v$ &.$/ 14$ &.$/ M$ & وزن هزار دانه (گرم) \\
\hline$-.1 \cdot 0$ & 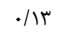 &.$/ 98$ & .1 .4 & $\overline{-.1 .9}$ & طول دوره ير شدن دانه \\
\hline . & -.1 .9 & $. \cdot . / \Delta F$ & .1 .9 &.$/ N r$ & سرعت ير شدن دانه \\
\hline.$/ \Delta F$ &.$- / . r$ &.$/ .1$ &.$/ V T$ & $\overline{. / \cdot \Delta}$ & عملكرد دانه (تن) \\
\hline$\overline{. / 94}$ &.$- / \cdot r$ & 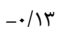 & $\overline{. / 4 q}$ & $-. .1+1$ & عملكرد بيولوزيك(تن) \\
\hline.$/ 14$ & $.1 . \varphi^{\leftarrow}$ &.$/ .9$ &.$/ \mathrm{NH}^{\mathrm{C}}$ & .1 .91 & شاخص برداشت \\
\hline$r / \varphi_{\Delta}$ & $r / \cdot r$ & $T / V q$ & $r / \cdot r$ & $1 / 99$ & مقدار ويثه \\
\hline$r \cdot / T V$ & Is/NA & $|Q / Q|$ & $11 / 49$ & $11 / \cdot V$ & درصد واريانس \\
\hline$r \cdot / T V$ & rv/I & $\Delta T / G T$ & $8 \pi / 9 \Delta$ & $V \Delta / \cdot T$ & درصد تجمعى واريانس \\
\hline
\end{tabular}

طول دوره پر شدن دانه، عملكرد دانه و شاخص برد برداشت

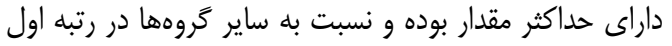

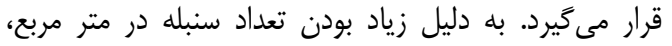

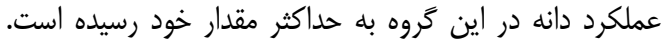

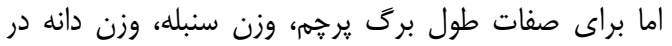

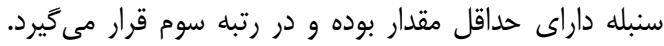

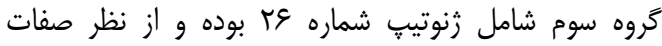

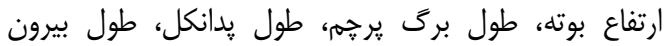

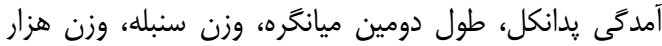

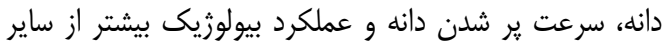

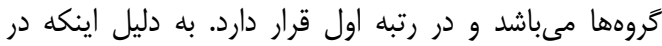

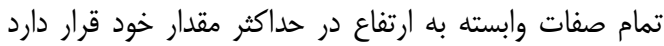

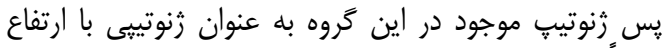

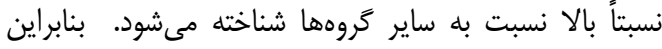

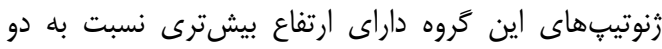

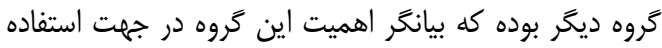

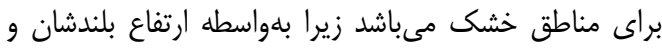

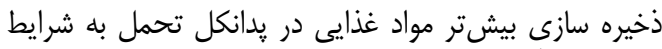

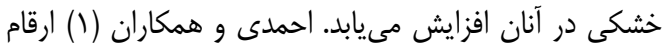

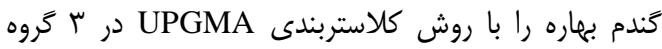

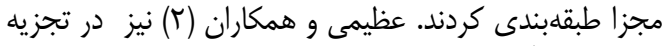

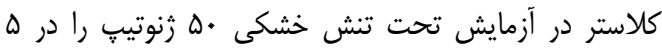

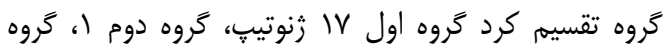

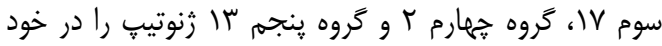

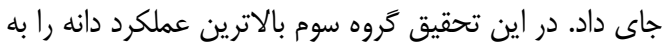

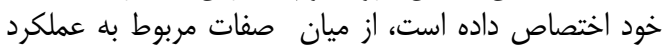

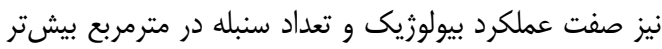

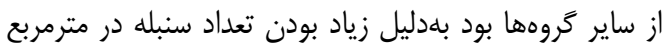

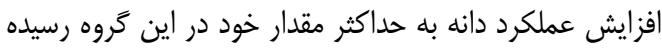

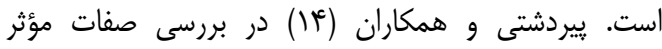

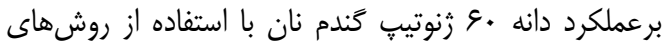

زنوتيڤهاى موجود در هر گَنه گَياهى داراى تنوع زيادى

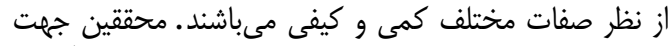

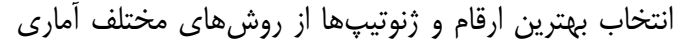

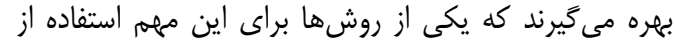

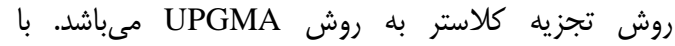

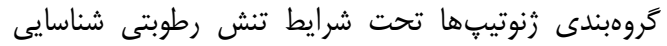

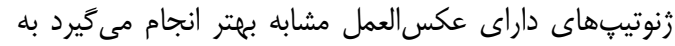

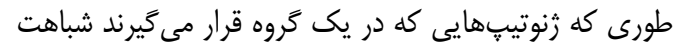

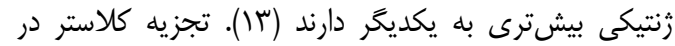

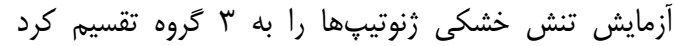

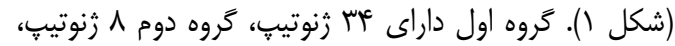

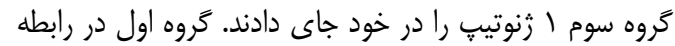

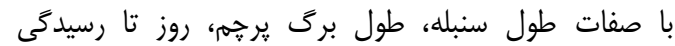

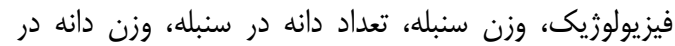

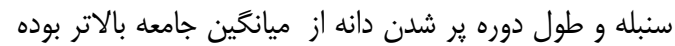

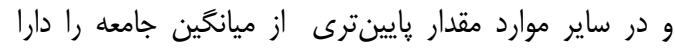

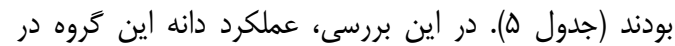

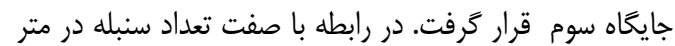

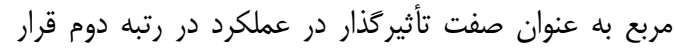

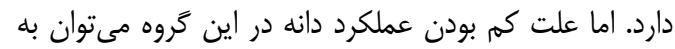

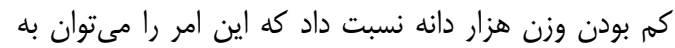

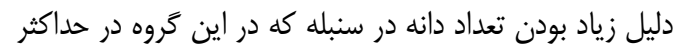

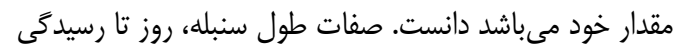

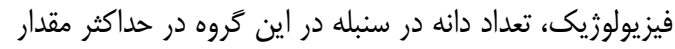

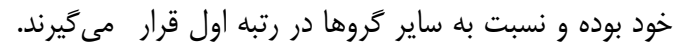

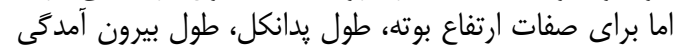

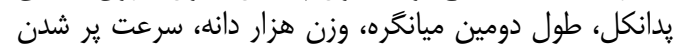

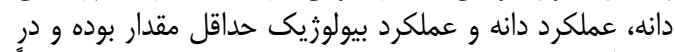

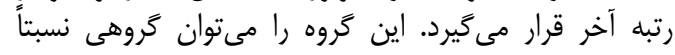

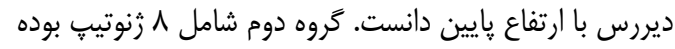

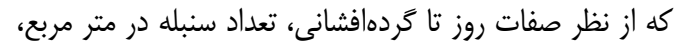


داراى قطر ساقه، تعداد دانه پِر شده و وزن هزار دانه بالا ميسر

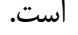

$$
\text { تشكر و قدردانى }
$$

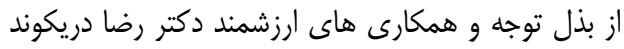

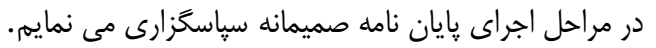

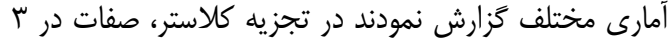

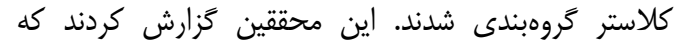

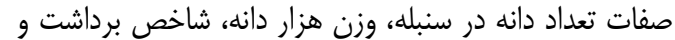

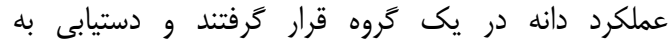

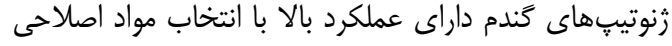

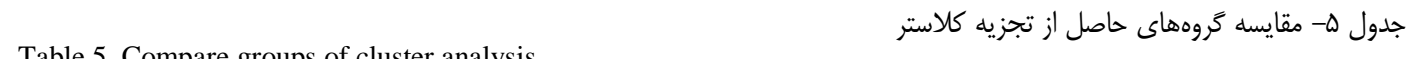

\begin{tabular}{|c|c|c|c|c|}
\hline ميانكَين جامعه & $\mu$ & r & 1 & صفات \\
\hline $99 / 14$ & AV/FY & $99 / 9$. & $99 / \mu$ & ارتفاع بوته (سانتىمتر) \\
\hline $1 . / 9 \mathrm{~V}$ & צ צ/r & १/१८ & $1 . / \mathrm{VV}$ & طول برك يرجهم (سانتى متر) \\
\hline$q / \% q$ & $\mathrm{~V} / \mathrm{TV}$ & T/r & $q / F v$ & طول سنبله (سانتىمتر) \\
\hline$\pi / \mu^{4}$ & $\mathrm{rq} / \mathrm{Av}$ & זו/ & r & طول پِدانكل (سانتىمتر) \\
\hline 1./9 & $T Y / \Delta V$ & $11 / . r$ & $1 \cdot / \Delta V$ & طول بيرون آمدگى بدانكل (سانتىمتر) \\
\hline $10 / 1 T$ & $\mid V / 1$. & $10 / v \Lambda$ & $16 / 91$ & طول دومين ميانكره (سانتىمتر) \\
\hline$I T / \Delta \Delta$ & $|r|$ & ITT/VF & $I T / \Delta Q$ & روز تا گردهافشانى \\
\hline $\mid V q / A f$ & IVG/ & IVa/Va & IVa/ar & روز تا رسيدگى فيزيولوزيك \\
\hline IVE/gT & $11 T / 9 V$ & rIS/IT & $199 / \pi T$ & تعداد سنبله در متر مربع \\
\hline$T / V I$ & $r / 19$ & $T / F^{2}$ & T/VQ & وزن سنبله (كرم) \\
\hline 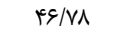 & f.199 & FT/KT & $F N \cdot \Delta$ & تعداد دانه در سنبله \\
\hline 1/99 & $T / Y \Lambda$ & $1 / \lambda$. & 1/99 & وزن دانه در سنبله (كرم) \\
\hline$F r / 99$ & $\Delta \mathrm{V} / \mathrm{V} \omega$ & $F / \mathrm{KI}$ & $\mathrm{FT/.1}$ & وزن هزار دانه (كرمج9 \\
\hline$\Delta V / \uparrow \wedge$ & $\Delta F$ & $\Delta \mathrm{V} / \Delta \mathrm{F}^{\mathrm{C}}$ & $\Delta \mathrm{V} / \Delta \mathrm{Q}$ & طول دوره ير شدن دانه \\
\hline$\cdot / V^{\pi}$ & $1 / \cdot 1$ & $\cdot /$ Va & $\cdot / N T$ & سرعت بر شدن دانه \\
\hline T/T & T/VA & T/Vq & $T / T$. & عملكرد دانه (تن) \\
\hline N/99 & $11 / 19$ & $q / \Delta r$ & N/rq & عملكرد بيولوزيك (تن) \\
\hline$r \varepsilon / \Lambda$. & $r F / v q$ & $r q / T r$ & TE/TV & شاخص برداشت \\
\hline
\end{tabular}

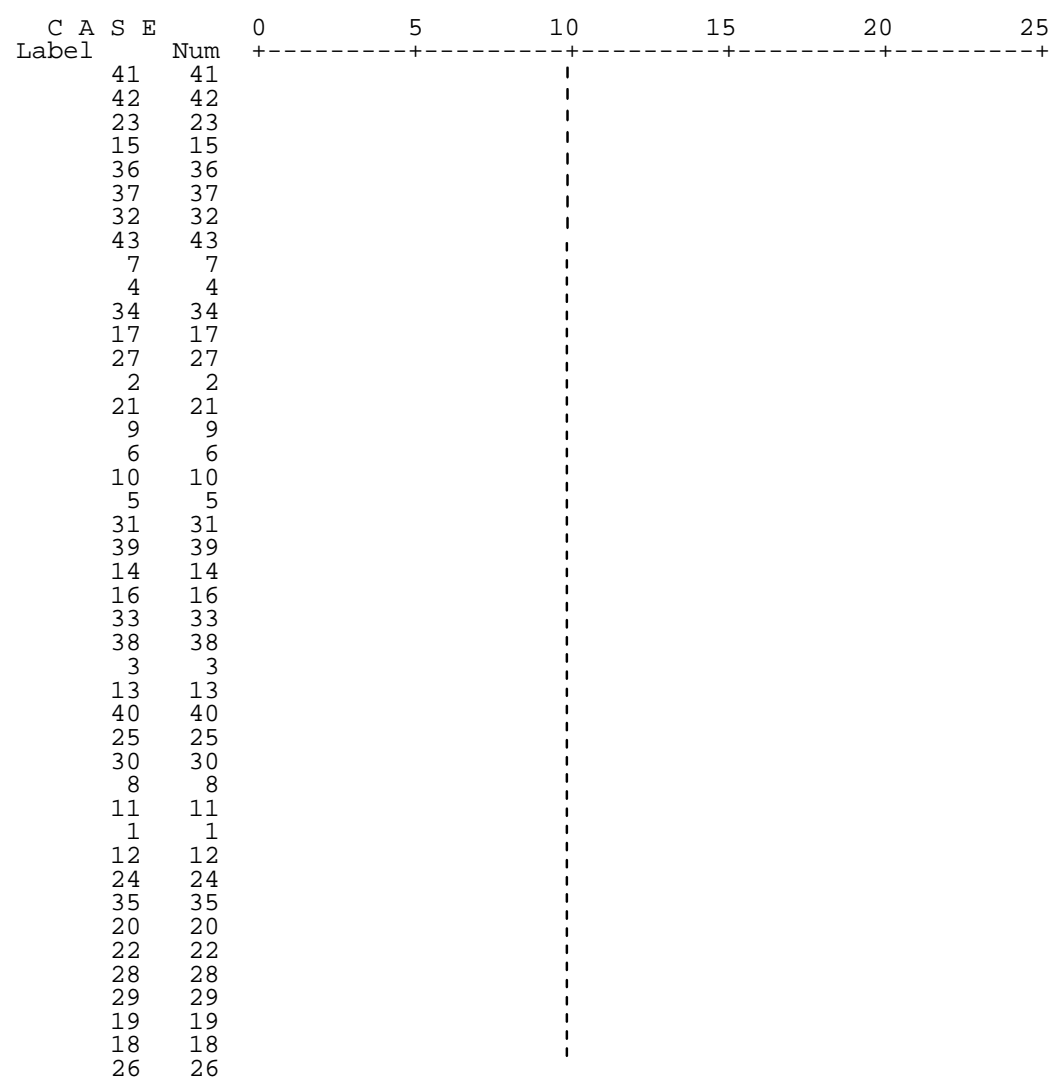

شكل ا- گروهبندى زنوتيبهاى گَندم براساس كليه صفات حاصل از تجزيه كلاستر

Figure 1. Grouping of genotypes wheat based on studied traits of cluster analysis 
1. Ahmadi, J. Mhatibi, H. Amirshekari and M. Amini Dehag 2012. Evaluation of the effec morpho-physiological indices on the yield of spring wheat (Triticum aestivum L.) using multivariate statistical methods. Journal of Agronomy Science, 4: 55-66 (In Persian).

2. Azimi, M. Study of plant traits related to terminal drought tolerance and dry matter remobilization in bread wheat lines. 2011. M.Sc. Thesis. Islamic Azad University Karaj Branch. 268 pp.

3. Blum, A. 1988. Plant Breeding for Stress Environments CRC Press, Inc. Boca Raton, Florida, USA, pp: $38-78$.

4. Babaei Zarch, M.J., M.H. Fotokian and S. Mahmoudi. 2014. Evaluation of Genetic Diversity of Wheat (Triticum Aestivum L.) Genotypes for Morphological Traits using Multivarite Analysis Methods, Journal of Crop Breeding, 6: 1-14.

5. Chaves, M.M. and M.M. Olivera. 2004. Mechanisms underlying plant resilience to water deficits: prospects for water-saving agriculture. Journal of Experimental Botany, 55: 2365-2384.

6. Evans, L.T. and R.A. Fischer. 1999. Yield potential. Crop Science, 39: 1544-1551.

7. Fischer, R.A. and R. Maurer. 1978. Drought resistance in spring wheat cultivars. I. Grain yield responses. Australian Journal of Agricultural Research, 29: 897-912.

8. Guttieri, M.J., J.C. Stark, K. O'B rien and E. Souza. 2001. Relative sensitivity of spring wheat grain yield and quality parameters to moisture deficit. Crop Science, 41: 327-335.

9. Gupta, N.K., S. Gupta and A. Kumar. 2001. Effect of water stress on physiological attributes and their relationship with growth and yield of wheat cultivars at different stages. Journal of Agronomy and Crop Science, 186: 55-62.

10. Heidari, B., G. Saeidi and B. Sayed-Tabatabaei. 2008. Factor analysis for quantitative traits and path analysis for grain yield in wheat. Water and Soil Science Journal, 11: 135-143.

11. Ludlow, M.M. and R.C. Muchow. 1990. A critical evaluation of improving crop yield in waterlimited environments. Advances in Agronomy, 43: 107-153.

12. Moghaddam, M., B. Ehdaie and J.G. Waines. 1997. Genetic variartion and interrelationships of agronomic characters in landraces of bread wheat from southeastern Iran. Euphytica, 95: 361-369.

13. Maleki Nejad, R. and M.M. Majidi. 2015. Evaluation of Iranian and Foreign Safflower Germplasms under Normal and Drought Stress Conditions. Journal of Crop Breeding, 7: 1-15.

14. Pirdashti, H., A. Ahmadpour, F. Shafaati, S. Hosseini, A. Shahsavari and A. Arab. 2012. Evaluation of most effective variables based on statistically analysis on different wheat genotypes. International Journal of Agriculture: Research and Review, 2: 381-388.

15. Rostae, M., D. Sadeghzadeh and U. Arshad. 2003. Study of relationship among grain yield agronomic traits using factor analysis in rainfed conditions. Journal of Agriculture Science, 13: 110(In Persian ).

16. Rajaram, S., H.J. Braun and M. Ginkel. 1996. CIMMYT's approach to bread for drought tolerance. Eupgytica, 92: 147-153.

17. Rosielle, A.T. and J. Hamblin. 1981. Theoretical aspects of selection for yield in stress and non-stress enviroments. Crop Science, 21:943-945

18. Stone, P. J. and M.E. Nicolas. 1995. A survey of the effects of high temperature wheat cultivars. Australian Journal of Agricultural Research, 46: 475-492.

19. Tousi Mojarad, M., M.R. Ghanadha and M. Khodarahmi. 2005. Factor analysis for grain yield and other attributes in bread wheat. Pajoush and Sazandegi, 67: 9-16 (In Persian).

20. Zakizadeh, M., M. Esmaeilzadeh and D. Kahrizi. 2010. Study on genetic variation and relationship between plant characteristics and grain yield in long spike bread wheat (Triticum aestivum L.) genotypes-using multivariate analysis. Iranian Journal of Crop Sciences, 12: 18-30 (In Persian).

21. Zhong-hu, H. and S. Rajaram. 1993. Differential responses of bread wheat characters to high temperature. Euphytica, 72: 197-303. 


\title{
Evaluation of Different Characteristics of wheat Genotypes under Drought Stress using Multivariate Statistical
}

\author{
Azam Rahimi Chegeni ${ }^{1}$, Mohammad Reza Bihamta ${ }^{2}$ and Manoocher Khodarahmi ${ }^{3}$ \\ 1- Graduated M.Sc., Plant Breeding, Department of Plant Breeding, Karaj Branch, Islamic Azad University, Karaj, \\ Iran (Corresponding Author: rahimiazam20@ yahoo.com) \\ 2- Professor, Department of Agronomy and Plant Breeding, College of Agriculture and Natural Resources, Tehran \\ University, Karaj, Iran \\ 3- Assistant Professor, Seed and Plant Improvement Institute, Karaj, Iran \\ Received: May 24, $2015 \quad$ Accepted: November 1, 2015
}

\begin{abstract}
In order to evaluate and grouping wheat genotypes based on grain yield and important agronomic traits under drought stress, a field experiment was conducted with 43 wheat genotypes using a Randomized Complete Block design in three replication at Azad University, khorramabad, Iran in 2012-2013 cropping season. Evaluated traits were: day to maturity, day to pollination, grain filling rate, grain filling period, plant height, second internode length, peduncle length, peduncle extrusion, spike length, flag leaf length, spike weight, grain per spike, harvest index, thousand weight kernel, grain weight per spike, spike per square meter, biological yield, grain yield. Analysis of variance showed that there were significant difference among genotypes for most traits under drought stress. Factor analysis based on principle components and varimax rotation showed that 5 factors, yield, growth characteristics, spike index and factor affecting of grain characteristics explained $75.02 \%$ of data total variance under drought stress. Lastly cluster analysis based on UPGMA method was used for grouping genotyprs and genotypes were divided into 3 groups. The first group was the third place for grain yield. The second group was consist of 8 genotype and the maximum grain yield was compared to the other groups. 35, 18,19,24 genotypes showed the highest yield. The third group was in all related traits height the maximum amount.
\end{abstract}

Keywords: Cluster analysis, Drought Stress, Factors analysis, Wheat 